\title{
Parametric Resonance in Dissipative Field Dynamics
}

\author{
Rudnei O. Ramos* and R. A. S. Vartuli ${ }^{\dagger}$ \\ Departamento de Física Teórica, Universidade do Estado do Rio de Janeiro, 20550-013 Rio de Janeiro, RJ, Brazil
} (Received on 14 Abril, 2008)

\begin{abstract}
The effects of bosonic trilinear interactions are investigated in the process of resonant particle production. We study a generic model of three fields with trilinear couplings. In this model a scalar field $\phi$ can excite a heavy bosonic field $\chi$ which then decays into a light bosonic field $\sigma$. We study the consequences of dissipation and stochastic noise terms in the field equations of motion that result from a calculation in nonequlibrium quantum field theory and determine the effects of these terms for the phenomenon of parametric resonance, which can occur e.g. during preheating after inflation.
\end{abstract}

Keywords: Parametric resonance; Particle production; Nonequlibrium dynamics

\section{INTRODUCTION}

Nonequilibrium dynamics is expected to happen in many pressing topical phenomena, including, for example, heavyion collisions and cosmology. In the context of cosmology, we have interest in understanding processes like particle production in the early universe and the physics of preheating after inflation. In the context of heavy-ion collisions, the recent experiments in the RHIC concerning the possibility of formation of a quark-gluon plasma, the study and understanding of the processes involved require the use of nonequilibrium quantum field theory methods.

Nonequilibrium dynamics involve complex processes like radiation/particle production, dissipation and stochastic dynamics. Dissipative processes may lead to important phenomenological consequences. For example, it is well understood today that models based in inflation generally present dissipative effects during the inflationary period [1]. These effects may have two important consequences for the inflationary dynamics. In the first place, they result in production of radiation during inflation and can influence the generation of fluctuations that serve to generate large-scales structures. Secondly, which is also true for other nonequilibrium processes, dissipative processes are related to time non-local terms in the effective evolution equation of the system (for example, for a background scalar field describing the system, like the inflaton field or a order parameter for a phase transition problem). Both dissipation and stochastic (noise) terms can influence significantly the nature and the history of the overall dynamics. For example, in the context of dissipation in inflationary models, it has lead to the distinction of inflation in two dynamical possibilities known as the cold inflation and warm inflation. In the cold inflation picture the inflationary expansion occurs with the universe in a supercooled phase which subsequently ends with a reheating period that introduces radiation into the universe. The fluctuations created during inflation are effectively zero-point ground state fluctuations and the evolution of the inflaton field is governed by a ground state evolution equation. The other picture of infla-

\footnotetext{
*Electronic address: rudnei@uerj.br

$\dagger$ Electronic address: rodvartuli@bol.com.br
}

tion dynamics is nonisentropic or warm inflation [1, 2]. In this picture, the inflationary expansion and radiation production occur concurrently. Moreover, the fluctuations created during inflation emerge from some excited statistical state and the evolution of the inflaton has dissipative terms arising from the interaction of the inflaton with other fields.

In this work, we are interested in studying how dissipative and stochastic (noise) terms, generally present in nonequilibrium dynamics, can influence particle production through the process of parametric resonance [3]. In the process of parametric resonance an oscilatting background field can induce particle production when coupled to other field through a resonance process, by which fluctuations of the field coupled to the background field can grow to very large values. How dissipation and stochastic terms influence the process of particle production through parametric resonance is an important issue. At one side we expect that dissipative effects may work against this process by damping not only the background field oscillations, but also fluctuations of any other fields coupled to it. But on the other hand, stochastic (or noise) terms may lead to an increase of particle production by raising the fluctuations amplitude. Both dissipation and noise terms may have significative influence, for example, in the process of preheating after inflation, whose leading dynamical process is through parametric resonance. For models based on the warm inflation, where, in this case the effects of the fluctuation and dissipation are their characteristic feature, a preheating, or parametric resonance phase after inflation in these models may lead to distinct consequences than those of the cold inflation picture.

In this work we determine how the presence of the fluctuation and dissipation terms affect the parametric resonance and the particle production processes. We present analytical and numerical results obtained for the dynamic equations found through the use of the formalism of quantum field theory in real time.

\section{EFFECTIVE EQUATIONS OF MOTION FOR COUPLED FIELDS}

We consider the model described by the Lagrangian density 


$$
\begin{aligned}
\mathcal{L} & =\frac{1}{2}\left(\partial_{\mu} \phi\right)^{2}-\frac{1}{2} m_{\phi}^{2} \phi^{2}+\frac{1}{2}\left(\partial_{\mu} \chi\right)^{2}-\frac{1}{2} m_{\chi}{ }^{2} \chi^{2}-g \phi \chi^{2} \\
& +\frac{1}{2}\left(\partial_{\mu} \sigma\right)^{2}-\frac{1}{2} m_{\sigma}^{2} \sigma^{2}-h \chi \sigma^{2}
\end{aligned}
$$

In Eq. (1), $\phi$ will represent a background field whose dynamics can influence that of the $\chi$ field, considered to be the fluctuating field, while $\sigma$ is a radiation bath field, representing the decay products of the $\chi$ field. From the trilinear coupling $g \phi \chi^{2}$, the field $\phi$ can decay directly into $\chi$ particles. Though $\phi$ does not decay directly into $\sigma$, it can still transfer its energy to $\sigma$ through its coupling to the $\chi$ field. Consequently, we can have dissipative effects on both the $\phi$ and $\chi$ effective equations of motion. The use of only trilinear couplings here is motivated by the fact that there is a much more transparent description of dissipation when interaction terms are of the trilinear form, in which case dissipation can readily be associated with the typical decay widths allowed (see below). This is well known in the cosmology literature about the old reheating scenario, used e.g. after preheating, to describe the thermalization phase afterwards [3]. Besides this, at leading order in the dissipative effects, all equations of motion become linear, which then allow at least some analytical work compared to the case where, e.g., quadratic couplings are also allowed. So, we here only restrict our analysis to trilinear couplings.

An explicit derivation of the effective equations of motion for the field $\phi$ as well as for the fluctuation field $\chi$ (see for example Refs. [2, 4] for details on these derivations) lead to the resulting equations of motion for $\phi$ and $\chi$, respectively,

$$
\begin{aligned}
& \left(\square+m_{\phi}^{2}\right) \phi_{c}(x)+\int_{-\infty}^{t} d t^{\prime} \int d^{3} x^{\prime} C_{\chi}\left(x-x^{\prime}\right) \phi_{c}\left(x^{\prime}\right)=\xi(x),(2) \\
& \left(\square+m_{\chi}^{2}+g \phi_{c}\right) \chi_{c}(x)+\int_{-\infty}^{t} d t^{\prime} \int d^{3} x^{\prime} C_{\sigma}\left(x-x^{\prime}\right) \chi_{c}\left(x^{\prime}\right)=\eta(x)(3)
\end{aligned}
$$

where $\xi(x)$ and $\eta(x)$ are Gaussian stochastic noise terms with two-point correlation functions that can be expressed in terms of the $C_{\phi}$ and $C_{\chi}$ kernels appearing in these equations [5, 6]. The equations for $\phi$ and $\chi$ are easily to be dealt with by taking the spacial Fourier transform for them. We then find, e.g., for Eq. (2):

$\ddot{\phi}_{k}(t)+\left(k^{2}+m_{\phi}^{2}\right) \phi_{k}(t)+\int_{-\infty}^{t} d t^{\prime} C_{\chi, k}\left(t-t^{\prime}\right) \phi_{k}\left(t^{\prime}\right)=\xi_{k}\left(t^{\prime}\right)$

Now, we take the time Fourier transform of (4) and solving it for the zero mode, $k=0$, which corresponding to the case of an homogeneous background field (like for example the inflaton or an homogeneous order parameter), we obtain for $\phi_{k=0}(t)$ the solution [7],

$$
\begin{aligned}
\phi(t) & =\phi\left(t_{i}\right) e^{-\frac{1}{2} \tilde{\Gamma}\left(m_{\phi}\right)\left(t-t_{i}\right)} \cos m_{\phi}\left(t-t_{i}\right) \\
& +\frac{\dot{\phi}\left(t_{i}\right)}{m_{\phi}} e^{-\frac{1}{2} \tilde{\Gamma}\left(m_{\phi}\right)\left(t-t_{i}\right)} \sin m_{\phi}\left(t-t_{i}\right) \\
& +\frac{1}{m_{\phi}} \int d t^{\prime} e^{-\frac{1}{2} \tilde{\Gamma}\left(m_{\phi}\right)\left(t-t^{\prime}\right)} \sin m_{\phi}\left(t-t^{\prime}\right) \xi_{0}\left(t^{\prime}\right),
\end{aligned}
$$

where $\tilde{\Gamma}\left(m_{\phi}\right)$ is the decay width of the $\phi$ field due to the decay channel $\phi \rightarrow \chi+\chi$, given by $[1,2]$

$\tilde{\Gamma}\left(m_{\phi}\right)=\frac{g^{2}}{8 \pi m_{\phi}}\left(1-\frac{4 m_{\chi}^{2}}{m_{\phi}^{2}}\right)^{1 / 2}\left[1+2 n_{B}\left(m_{\phi} / 2\right)\right] \theta\left(m_{\phi}-2 m_{\chi}\right)$,

and $n_{B}$ is the Bose distribution function and $\theta(x)$ is the step function.

In principle, a solution can also be obtained for $\chi$ from its effective equation of motion Eq. (3). Here, however, we cannot use the same approach used in the solution for $\phi$, since now the frequency of oscillation is not constant, but it is time variable due to the dependence on $\phi$. In this case we can use the approach developed in [8], where we can write a solution for $\chi(\mathbf{k}, t)$, with the initial conditions satisfying

$$
i \dot{\chi}(\mathbf{k}, 0)=\omega_{k} \chi(\mathbf{k}, 0), \quad|\chi(\mathbf{k}, 0)|=\frac{1}{\sqrt{2 \omega_{k}}},
$$

and resulting for the square of the amplitudes of $\chi$ and $\dot{\chi}$ to be given by

$$
\begin{aligned}
|\chi|^{2} & =\frac{e^{-\Gamma_{\chi} t}}{2 \omega_{k}}\left[\cosh (2 \mu t)-\sinh (2 \mu t) \sin \left(2 \omega_{k} t\right)\right], \\
|\dot{\chi}|^{2} & =\frac{e^{-\Gamma_{\chi} t}}{2 \omega_{k}} \omega_{k}^{2}\left[\cosh (2 \mu t)+\sinh (2 \mu t) \sin \left(2 \omega_{k} t\right)\right],
\end{aligned}
$$

where $\Gamma_{\chi}$ is the decay width of $\chi$ in the $\sigma$ particles, $\omega_{k}^{2}=$ $k^{2}+m_{\chi}^{2}$ and $\mu=m_{\phi}^{-1} \sqrt{g^{2} \Phi^{2}-\Delta^{2}}$, where $\Phi$ is the amplitude of the scalar $\phi$ field and $\Delta=\omega_{k}^{2}-m_{\phi}^{2} / 4$.

From Eq. (8), we can determine the evolution of the energy density of the $\chi$-particle with wavenumber $k, \rho_{\chi}^{k}$ as given by

$$
\begin{aligned}
\dot{\rho}_{\chi}^{k} & =\frac{d}{d t}\left(\frac{1}{2}|\dot{\chi}|^{2}+\frac{1}{2} \Omega_{k}^{2}|\chi|^{2}\right) \\
& =\frac{1}{2} \frac{d\left(\Omega_{k}^{2}\right)}{d t} \frac{1}{2} \Omega_{k}^{2}|\chi|^{2}-\Gamma_{\chi} \frac{1}{2} \Omega_{k}^{2}|\chi|^{2} \\
& \cong \frac{g \Phi}{m_{\phi}} \rho_{\chi}^{k}-\Gamma_{\chi} \rho_{\chi}^{k},
\end{aligned}
$$

where $\Omega_{k}^{2}=\omega_{k}^{2}+2 g \phi_{c}$ and we used Eq. (8) in the second line in (9), and in the third line of this same equation, we have considered the average of an oscillation period of the background field $\phi$. The first term corresponds to the usual resonant production due to the $\phi$ decay, while the second term shows us the decreasing of energy as a result of the decay of the $\chi$ particles. We can note that the energy density for the $\chi$-particles with wavenumber $k$ is reduced by a factor $e^{-\Gamma_{\chi} t}$. If we sum over all $k$, we obtain the total energy density for the resonant production of $\chi$-particles. The width of the bands of resonance is found to decrease because of dissipation, and it is given now by

$$
\mu \simeq \frac{g \Phi}{m_{\phi}} \rightarrow \frac{g \Phi}{m_{\phi}} \sqrt{1-\left(\frac{m_{\phi} \Gamma_{\chi}}{2 g \Phi}\right)^{2}}
$$


Analogous result can be determined if we consider for example the case of particle production after inflation and if $\Gamma_{\chi}<g \Phi / m_{\phi}(\simeq \mu)$. In this case, we find that the rate of the particle production can be expressed as

$$
\dot{\rho}_{\chi} \simeq \frac{H}{2 \pi^{2}}\left(\frac{m_{\phi}}{2}\right)^{2} \frac{1}{4} \exp \left[\frac{4 g^{2} \Phi^{2}}{H m_{\phi}^{3}}\left(1-\frac{2 m_{\phi} \Gamma_{\chi}}{2 g \Phi}\right)\right],
$$

where $H$ is the Hubble factor. Again, from Eq. (11) we see that dissipation tends to decrease the rate of particle production.

So far we have derived an analytical solution for the effective equation of motion for the $\chi$-field taking into account only the case without stochastic fluctuations, given by the $\xi$ and $\eta$ terms in the right-hand-side of Eqs. (2) and (3). Solutions for the case where the fluctuations (noise terms) are included are a much more complicate problem. However, we can demonstrate, numerically, that the existence of stochastic terms in the field equations of motion do not affect qualitatively the resonance bands as predicted by the Matthieu equation in the absence of noise [9]. We present below the numerical results obtained for the effective equations of motion, from a three-dimensional lattice simulation for the complete Langevin equations represented by Eqs. (2) and (2).

\section{NUMERICAL RESULTS FOR THE EFFECTIVE EQUATION OF MOTION}

Let us now present the numerical results obtained by lattice simulations for the effective equation of motion. We compare the results obtained from the classical equations of motion without dissipation and stochastic fluctuation and the case that includes dissipation and stochastic fluctuation. For all simulations shown here, we use the program Latticeasy [10] adapted to include dissipation and noise terms.

The trilinear couplings $g$ and $h$ (with mass dimension) are normalized as $g=\bar{g} m_{\phi}$ and $h=\bar{h} m_{\phi}$. In our simulations we have also included small quadratic interaction terms of the form $\frac{\lambda^{2}}{2} \phi^{2} \chi^{2}$ and $\frac{\alpha^{2}}{2} \chi^{2} \sigma^{2}$ for numerical stability. All parameters used in the simulation are then given by $\left(M_{\mathrm{Pl}}\right.$ is the Planck mass)

$$
\begin{aligned}
& m_{\phi}=10^{-6} M_{\mathrm{Pl}} \\
& m_{\chi}=10^{-7} M_{\mathrm{Pl}} \\
& m_{\sigma}=10^{-8} M_{\mathrm{Pl}} \\
& \bar{g}=10^{-7} \\
& \bar{h}=10^{-5} \\
& \lambda^{2}=\bar{g}^{3 / 2} \\
& \alpha^{2}=100 \bar{g}^{3 / 2} \\
& \phi_{0}=0.193 M_{\mathrm{Pl}} .
\end{aligned}
$$

The simulations were realized in a cubic lattice $\left(N^{3}\right)$ with $N=$ 32 , and of size $L=16$, which then gives a stepsize in space of $\Delta x=L / N=0.5$. The stepsize in time used was $\Delta t=0.01$ (in dimensionless units). In the lattice the noise terms were defined as

$$
\xi_{j, m}=\sqrt{\frac{2 \Gamma_{i} T}{\Delta x^{3} \Delta t}} G_{j, m}
$$

where $j$ e $m$ are spacial and temporal indices and $G_{j, m}$ is taken from a zero-mean unit-variance Gaussian.

The parameters given by Eq. (12) are, for example, relevant in the studies of parametric resonance after inflation [3]. With the above choice of parameters we have that the leading terms are still the ones with trilinear couplings and then, we expect that the results derived in the previous section still to be valid, like the ones given by Eqs. (5) and (11). For the equation of motion for $\chi$, Eq. (3), it is simpler to use for it a phenomenological approximate local form that includes explicitly a decay term of $\chi$ in the $\sigma$ channel,

$$
\left(\square+m_{\chi}^{2}+g \phi_{c}\right) \chi_{c}(x)+\lambda^{2} \phi^{2} \chi+\Gamma_{\chi} \dot{\chi}_{c}(x)=\xi_{\chi}(x),
$$

where $\Gamma_{\chi}$ is the decay width of $\chi \rightarrow 2 \sigma$, given by $[2,4,7]$

$$
\Gamma_{\chi}=\frac{h^{2}}{8 \pi m_{\chi}}\left(1-\frac{4 m_{\sigma}^{2}}{m_{\chi}^{2}}\right)^{1 / 2}\left[1+2 n_{B}\left(m_{\chi} / 2\right)\right] \theta\left(m_{\chi}-2 m_{\sigma}\right)
$$

while for $\phi$, consistent with the solution (5), we consider

$$
\left[\square+m_{\phi}^{2}+\lambda^{2} \chi^{2}(x)\right] \phi(x)+g \chi^{2}(x)+\Gamma_{\phi} \dot{\phi}=\xi_{\phi}(x),
$$

with $\Gamma_{\phi}$ given by Eq. (6). The noise terms $\xi_{\phi}$ and $\xi_{\chi}$ in Eqs. (14) and (16) are chosen to satisfy the conditions of being Gaussian, $\left\langle\xi_{i}(x)\right\rangle=0, i=\phi, \chi$ and to have a two-point correlation function satisfying the fluctuation and dissipation theorem in the classical limit $[5,6]$,

$$
\left\langle\xi_{i}(x) \xi_{i}\left(x^{\prime}\right)\right\rangle=2 T \Gamma_{i} \delta^{3}\left(\mathbf{x}-\mathbf{x}^{\prime}\right) \delta\left(t-t^{\prime}\right)
$$

Note that though the fluctuation and dissipation theorem is an equilibrium expression at a temperature $T$, here we take the point of view that the dynamics of the system, although of nonequilibrium, can still be described locally as being at equilibrium with a temperature $T$ approximately given by the system's energy, according to the equipartition theorem, and chosen to be approximately $m_{\phi}$, which is typically the magnitude of the temperature of reheating for the system after thermalization.

Finally, for the $\sigma$ field we consider the equation

$$
\left[\square+m_{\sigma}^{2}+h \chi(x)+\alpha^{2} \chi^{2}(x)\right] \sigma(x)=0,
$$

without dissipation or noise terms, since we have not considered the possibility of $\sigma$ to decay (the final radiation bath is made of $\sigma$ particles). 


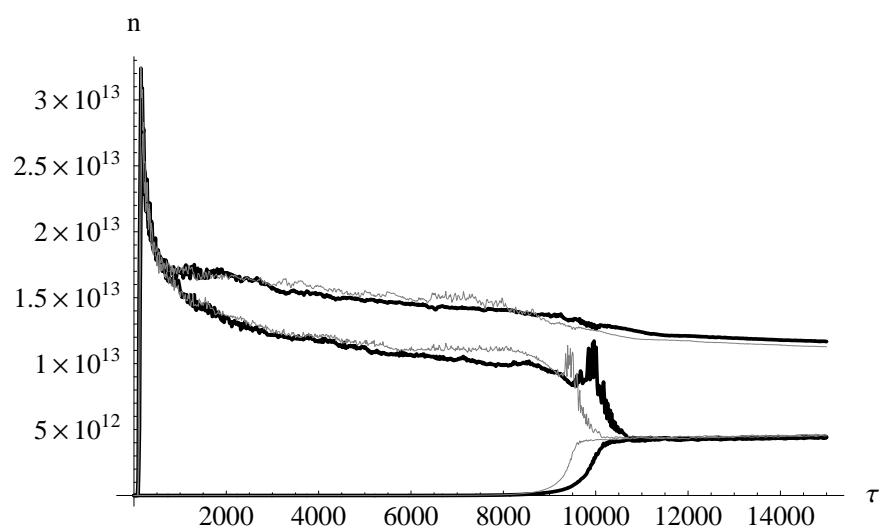

FIG. 1: Number Particle Density produced for the fields (from above to below) $\phi, \chi$ and $\sigma$, as a function of $\tau=m_{\phi} t$ in the case of the classical evolution for the fields with no dissipation or noise (gray) and including dissipation and noise (black).

We must note a difference in the effective equations studied here as compared to the case studied e.g. in Refs. [1, 2, 4-6], where a quasi-adiabatic regime was used. In those references dissipation is a higher order effect and direct decay is strongly suppressed. Here, on the other hand, we are in a fast oscillatory regime for the fields and the opposite is true, with dissipation coming dominantly from the decay rate directly (see e.g. Ref. [7]). This also justifies the use of the effective equations in a local form as in Eqs. (14) and (16).

In Fig. 1 we show the results for particle number density produced for each field due to parametric resonance, and defined by the formula $(i=\phi, \chi, \sigma)$

$$
n_{i}(t)=\int \frac{d^{3} k}{(2 \pi)^{3}} n_{k}(t)
$$

where $n_{k}(t)$, for $\chi$ and similarly for the other fields, defined by

$$
n_{k}=\frac{\omega_{k}}{2}\left(\frac{1}{\omega_{k}^{2}}\left|\dot{\chi}_{k}\right|^{2}+\left|\chi_{k}\right|^{2}\right)
$$

Figure 1 shows that the effect of dissipation and noise terms, though small here as a consequence of the parameter values used in our simulations, can still be expressive as compared with the simulations without these terms. In particular, we see the effects of a decrease of particle production through parametric resonance as predicted, e.g., by Eq. (10) or (11).

\section{CONCLUSIONS}

We could as well have included in the Lagrangian model coupling terms such as $\phi^{2} \chi^{2}$ or $\chi^{2} \sigma^{2}$. Alone, without trilinear terms, these interaction terms lead to a very different parametric resonance regime, with bands of resonance in parameter space dominated by narrow bands. In the presence of trilinear terms, however, the resonance bands change to broad resonance ones over a large part of the parameter space, when compared to the case of quadratic couplings. Quadratic couplings, tough more general, are more difficult to analyze analytically as compared to trilinear couplings, and so we have restricted our study here only to the case of trilinear couplings.

In conclusion, in this work we have studied the effective equations of motion for a background field, a fluctuation field and a radiation field. The equations are shown to exhibit the usual parametric resonance phenomenon, allowing for exponential amplification of the radiation modes inside the instability bands. In this work, we have presented the analytical and numerical results obtained for the dynamical equations for all the fields for the model studied. We have numerically seen that the fluctuation and dissipation terms can generate quantitative effects in the dynamics of the resonant particle productions due to the damping of fields oscillations and, consequently, a reduction in the rate of the particle production. In our numerical results, however, the effects were small due to the values of parameters that we have restricted our simulations, so to obtain stable numerical results within the time required to observe the effect of parametric resonance.

\section{Acknowledgments}

The authors would like to S. E. Joras for discussions. ROR was partially supported by CNPq. RASV was supported by CAPES and FAPERJ.
[1] A. Berera, M. Gleiser, and R. O. Ramos, Phys. Rev. D 58, 123508 (1998); A. Berera and R. O. Ramos, Phys. Lett. B 567, 294 (2003).

[2] A. Berera and R. O. Ramos, Phys. Lett. B567, 294 (2003).

[3] L. Kofman, A. Linde, and A. A. Starobinsky, Phys. Rev. Lett. 73, 3195 (1994); Phys. Rev. D 56, 3258 (1997); Y. Shtanov, J. Traschen, and R. Brandenberger, Phys. Rev. D D51, 5438 (1995).

[4] A. Berera and R. O. Ramos, Phys. Lett. B 607, 1 (2005); Phys. Rev. D 71, 023513 (2005).
[5] M. Gleiser and R. O. Ramos, Phys. Rev. D 50, 2441 (1994).

[6] A. Berera, I. G. Moss, and R. O. Ramos, Phys. Rev. D 76, 083520 (2007).

[7] J. Yokoyama, Phys. Rev. D 70, 103511 (2004).

[8] D. Y. Hsieh, J. Math. Phys. 21, 722 (1980).

[9] M. Gitterman, R. I. Shrager, and G. H. Weiss, Physics Lett. A 142, 84 (1989)

[10] Latticeeasy home page: http://www.science.smith.edu/departments/ Physics/fstaff/gfelder/latticeeasy/ 